\title{
The Generalized Bode Criterion: application to the de voltage control of a three-phase photovoltaic grid-tied inverter
}

\author{
David Lumbreras, Ernesto L. Barrios, Andoni Urtasun, Pablo Sanchis \\ Public University of Navarre (UPNA) \\ Institute of Smart Cities \\ Campus de Arrosadía \\ Pamplona, Spain \\ Email: david.lumbreras@unavarra.es
}

\section{Acknowledgements}

This work was supported by the Spanish State Research Agency (AEI) and FEDER-UE under grant DPI2016-80641-R and by the Public University of Navarre through a doctoral scholarship. The authors acknowledge the financial and continuous support of INGETEAM POWER TECHNOLOGY.

\section{Keywords}

$<<$ Converter control $>>,<<$ Frequency-Domain Analysis $>>,<<$ Photovoltaic $>>,<<$ Design $>>$

\begin{abstract}
As renewable energies are becoming more important in the electrical generation system, power electronic converters are facing new design issues related not only to their components but also to their control loops. In this context, the Generalized Bode Criterion (GBC) appears as a good tool to correctly determine stability and to help the controller design. In order to show the potential of the GBC and how it can be applied, this paper studies a dc voltage regulation with compensation of the photovoltaic power in a three-phase photovoltaic grid-tied inverter.
\end{abstract}

\section{Introduction}

The year 2017 has experienced the largest ever increase in renewable energy power, with a total installed power of $178 \mathrm{GW}$ [1]. Solar photovoltaic led the way with a new installed power of $98 \mathrm{GWp}$, which is more than the net additions of fossil fuels and nuclear power combined [1].

Due to the expansion of renewable energy systems, power electronic converters are becoming more important. The decreasing cost of these systems is creating new challenges in the converter design process related not only to their components but also to their control loops [2]. Particularly, the stability analysis of the control loops and the tuning of the controller are becoming important issues. The traditional Bode criterion and its revisions, which are easy to apply to the controller design, cannot be used to determine the stability of all power electronic applications since they can provide false information [2], [3]. The Nyquist criterion can always be applied, but it is not intuitive for the controller design process [2], [4]. The recently proposed Generalized Bode Criterion (GBC) [2] combines the advantages of the Bode and Nyquist criteria. The GBC can always be used to determine the stability of a control loop, since it is based on the Nyquist criterion, and it is easy to apply to the controller design process, since it uses information from the Bode diagram and the open-loop transfer function.

In this paper, an application which shows the potential of the Generalized Bode Criterion is presented. The studied system is a photovoltaic grid-tied inverter with an LC output filter such as the one shown 
in Fig. 1. Traditionally, a cascaded regulation is used in this application, where the outer dc-link voltage control loop provides the reference for the inner current control loops [5]. However, this control method requires large dc-link capacitance in order to avoid instability. This occurs whether the dc voltage, $v_{d c}$, or the square of the dc voltage, $v_{d c}{ }^{2}$, is controlled [6]. In particular, for $v_{d c}{ }^{2}$ regulation, the compensation of the photovoltaic power, $P_{p v}$, can be used as an alternative to reduce the capacitance value. The existing delays in the control loops, especially in high power converters, still make it very difficult to stabilize the $v_{d c}{ }^{2}$ regulation with this compensation. Therefore, this paper analyzes the stability of a $v_{d c}{ }^{2}$ regulation with compensation of the photovoltaic power by using the Generalized Bode Criterion, and proposes the addition of a lead compensator, which is designed applying the GBC.

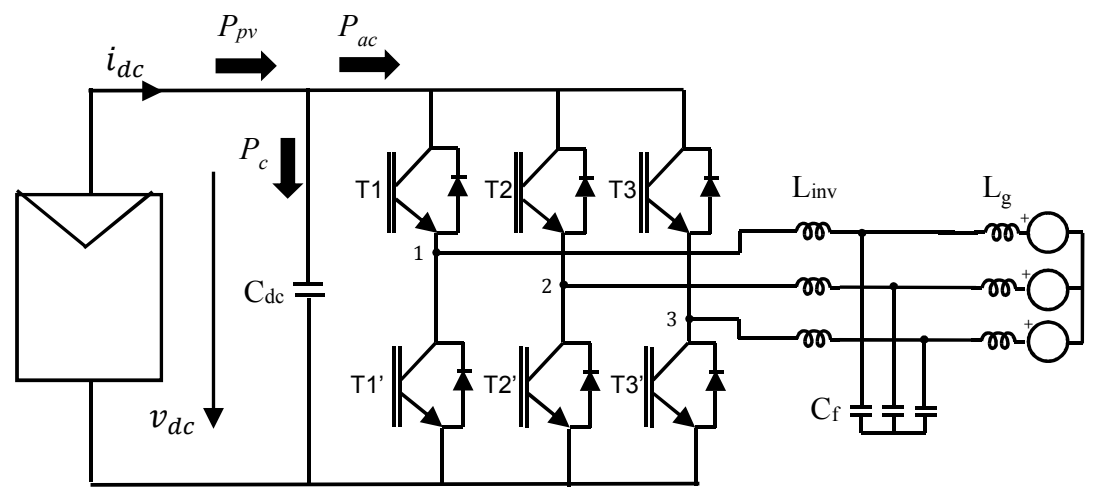

Fig. 1. Three-phase photovoltaic grid-tied inverter with LCL output filter.

A brief summary of the Generalized Bode Criterion is included in section II. The $v_{d c}{ }^{2}$ regulation is presented in section III. In section IV, the Generalized Bode Criterion is used to analyze the case study. First, thanks to the GBC, the need of lead compensator is demonstrated. Then, applying the GBC, the lead compensator is designed in order to ensure a stable regulation. Finally, the photovoltaic application is simulated in order to validate the stability conclusions obtained with the GBC and to demonstrate that the control method is stable in the whole operating range, making it possible to reduce the dc-link capacitance.

\section{The Generalized Bode Criterion (GBC)}

The Generalized Bode Criterion [2] is based on the Nyquist criterion, but it is calculated from the information of the Bode diagram and the open-loop transfer function, therefore facilitating the stability analysis. The Nyquist criterion is expressed as [7]:

$$
Z=P-N,
$$

where $Z$ is the number of poles in the right hand plane (RHP) of the closed-loop transfer function, $N$ the number of encirclements of the open-loop Nyquist diagram around the $(-1,0 \mathrm{j})$ point and $P$ the number of the open-loop RHP poles.

An easy way to count the number of encirclements around the $(-1,0 j)$ point, is to count the number of crossings of the Nyquist diagram with a half-line that starts at point $(-1,0 \mathrm{j})$, placed in the negative real axis direction [8]. As a consequence of using this half-line, the crossings are easy to identify in the Bode diagram since they occur at points with gain higher than $0 \mathrm{~dB}$ and phase $\pm n \cdot 180^{\circ}$, with $n$ an odd number. However, the Bode diagram is only defined for positive frequencies. If the transfer function coefficients are real, then the number of crossings produced at positive and negative frequencies is identical. Therefore, in order to obtain the total number of crossings, it is only necessary to calculate the crossings 
produced at a frequency of $0 \mathrm{~Hz}$. Using these equivalences, the Generalized Bode criterion is formulated as [2]:

$$
Z=P-\left[2 \cdot\left(C^{+}-C^{-}\right)+C_{0}\right],
$$

where $C^{+}$is the number of crossings in the Bode diagram of the open-loop transfer function with phase $\pm n \cdot 180^{\circ}$ (being $n$ an odd number), with gain higher than $0 \mathrm{~dB}$ and increasing phase; $C^{-}$is the number of crossings counted in the Bode diagram under the same conditions as $C^{+}$but with decreasing phase and $C_{0}$ represents the crossings under the same conditions but at zero frequency. $C^{+}$and $C^{-}$can easily be obtained by the Bode diagram and $C_{0}$ is calculated in [2] and now summarized in Table I for the most common cases in power electronics:

\begin{tabular}{c|c|c|c}
\hline \hline $\begin{array}{c}\text { Number of } \\
\text { integrators }(k)\end{array}$ & $\begin{array}{c}\text { de gain without integrators } \\
\left(K_{G}\right)\end{array}$ & Phase at $0^{+} \mathrm{Hz}\left(\varphi\left(0^{+}\right)\right)$ & $\begin{array}{c}\text { Crossings at zero } \\
\text { frequency }\left(C_{0}\right)\end{array}$ \\
\hline$k<0$ & - & - & 0 \\
\hline \multirow{3}{*}{$k=0$} & $K_{G}>-1$ & - & 0 \\
\cline { 2 - 4 } & $K_{G}<-1$ & $\varphi\left(0^{+}\right)<-180^{\circ} /$ decreasing phase & -1 \\
\cline { 2 - 4 } & & $\varphi\left(0^{+}\right)>-180^{\circ} /$ increasing phase & +1 \\
\hline \multirow{2}{*}{$k=1$} & $K_{G}>0$ & - & 0 \\
\hline \multirow{3}{*}{$k=2$} & $K_{G}<0$ & - & -1 \\
\cline { 2 - 4 } & \multirow{2}{*}{$K_{G}>0$} & $\varphi\left(0^{+}\right)<-180^{\circ} /$ decreasing phase & 0 \\
\cline { 2 - 4 } & $K_{G}<0$ & $\varphi\left(0^{+}\right)>-180^{\circ} /$ increasing phase & -1 \\
\hline \hline
\end{tabular}

Table I. Value of $C_{0}$ for the most common cases in power electronics [2]

\section{Case study modeling and $v_{d c}^{2}$ regulation}

The studied application was shown in Fig. 1, and the de voltage regulation scheme is now presented in Fig. 2, where subscript $f$ represents measured and filtered values, and superscript * represents reference values. As mentioned in the introduction, the square of the dc voltage, $v_{d c}{ }^{2}$, is the control variable. In order to improve the performance of the system and to reduce the value of the input capacitance, a compensation of the photovoltaic power, $P_{p v}$, is included in the voltage regulation.

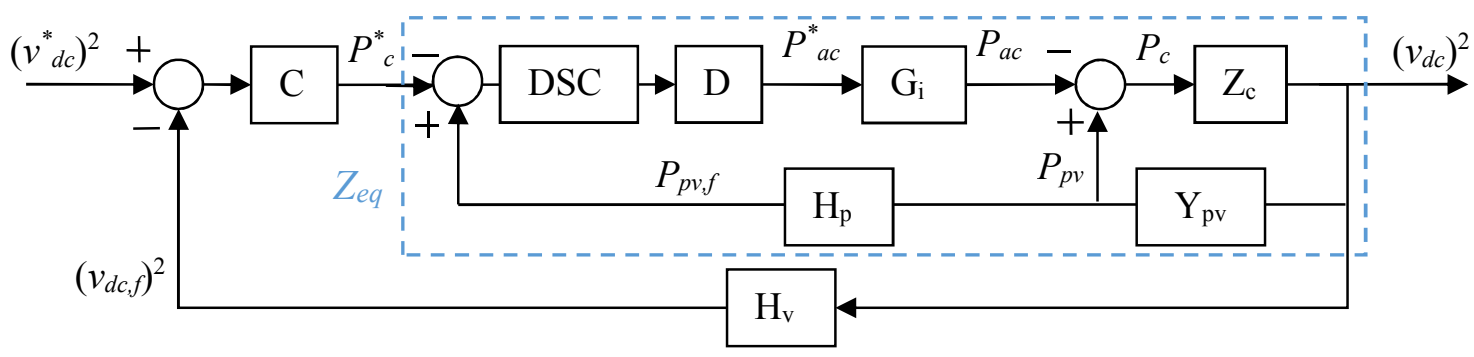

Fig. 2. Control loop for the studied $v_{d c}^{2}$ regulation.

All transfer functions from Fig. 2 are now studied. A delayed signal cancellation, $D S C$, is used to remove the second harmonic of the dc voltage, especially significant under asymmetric voltage sags. The DSC is modeled in the s-domain by applying Padé approximations with the necessary order to maintain accuracy [9]. If a $6^{\text {th }}$ order Padé approximation is used, the DSC transfer function is the following: 


$$
\begin{gathered}
\operatorname{DSC}(s)=\frac{1+e^{-5 \cdot T s}}{2} \approx \\
\approx \frac{\frac{3125}{133056} T_{s}^{6} \cdot s^{6}+\frac{4375}{5544} T_{s}^{4} \cdot s^{4}+\frac{875}{308} T_{s}^{2} \cdot s^{2}+1}{\frac{3125}{133056} T_{s}^{6} \cdot s^{6}+\frac{4375}{22176} T_{s}^{5} \cdot s^{5}+\frac{4375}{5544} T_{s}^{4} \cdot s^{4}+\frac{7875}{4158} T_{s}^{3} \cdot s^{3}+\frac{875}{308} T_{s}^{2} \cdot s^{2}+\frac{5}{2} T_{s} \cdot s+1}
\end{gathered}
$$

The combination of the digital sampling, computation and Zero-Order Hold delays is represented in Fig. 2 by $D$. Again, they are modeled in the s-domain with the necessary Padé approximations, obtaining in this case:

$$
D(s)=e^{-T_{s} \cdot s} \cdot \frac{1-e^{-T_{s} \cdot s}}{T_{s} \cdot s} \approx \frac{\frac{T_{s}^{2}}{12} \cdot s^{2}-\frac{T_{s}}{2} \cdot s+1}{\frac{T_{s}^{2}}{12} \cdot s^{2}+\frac{T_{s}}{2} \cdot s+1} \cdot \frac{1}{\frac{T_{s}^{2}}{12} \cdot s^{2}+\frac{T_{s}}{2} \cdot s+1},
$$

where the first term corresponds to the sampling and computation delays and the second term to the Zero-Order Hold delay.

$G_{i}$ represents the inner current control loops. In this application, the current is controlled in the synchronous reference frame with a PI controller. The closed-loop transfer function of the current control is modeled by a second-order transfer function, where the natural frequency, $\omega_{n}$, relates to the current control loop crossover frequency and the damping, $\xi_{i}$, to its phase margin.

$$
G_{i}(s)=\frac{1}{\frac{s^{2}}{\omega_{n}^{2}}+2 \cdot \frac{\xi}{\omega_{n}} \cdot s+1} .
$$

The capacitor impedance, $Z_{c}$, defined as the relationship between the square of the dc voltage, $v_{d c}^{2}$, and the power through the capacitor, $P_{c}$, can be obtained as follows:

$$
P_{c}=C_{d c} \cdot v_{d c} \cdot \frac{d v_{d c}}{d t}=\frac{C_{d c}}{2} \cdot \frac{d\left(v_{d c}^{2}\right)}{d t} \rightarrow Z_{c}(s)=\frac{v_{d c}^{2}}{P_{c}}=\frac{2}{C_{d c} \cdot s}
$$

The transfer function $Y_{p v}$ represents the relationship between the photovoltaic power, $P_{p v}$, and $v_{d c}{ }^{2}$, which can be obtained by means of a small-signal linearization of the generator P-V curve:

$$
\hat{P}_{p v}=\left.\frac{\partial P_{p v}}{\partial v_{d c}}\right|_{V_{d c}, I_{d c}} \cdot \hat{v}_{d c}=\left.\frac{1}{2 \cdot V_{d c}} \cdot \frac{\partial P_{p v}}{\partial v_{d c}}\right|_{V_{d c}, I_{d c}} \cdot \hat{v}_{d c}^{2}
$$

where $V_{d c}$ and $I_{d c}$ are the PV generator voltage and current at the operating point. Since $P_{p v}=v_{d c} \cdot i_{d c}$, where $i_{d c}$ is the PV current, (7) can be further developed as:

$$
\begin{aligned}
& \hat{P}_{p v}=-\frac{1}{2} \cdot\left(-\frac{\partial i_{d c}}{\partial v_{d c}}-\frac{I_{d c}}{V_{d c}}\right) \cdot \hat{v}_{d c}^{2}=-\frac{g_{p v}-g_{0}}{2} \cdot \hat{v}_{d c}^{2}=-\frac{g_{t}}{2} \cdot \hat{v}_{d c}^{2}, \\
& g_{t}=g_{p v}-g_{0}, g_{p v}=-\frac{\partial i_{d c}}{\partial v_{d c}}, \quad g_{0}=\frac{I_{d c}}{V_{d c}},
\end{aligned}
$$

where $g_{p v}$ is the dynamic conductance, $g_{0}$ the static conductance and $g_{t}$ the total conductance of the PV generator, which is positive above maximum power point (MPP), and negative below MPP. From the previous equations, $Y_{p v}$ can be expressed as: 


$$
Y_{p v}(s)=\frac{\hat{P}_{p v}}{\hat{v}_{d c}^{2}}=-\frac{g_{t}}{2} .
$$

The analog filters of the control loop are named with $H$. Both the voltage filter, $H_{v}$, and the power filter, $H_{p}$, are first-order low-pass filters with time constants $\tau_{v}$ and $\tau_{p}$ respectively. Their transfer functions are expressed respectively in (11) and (12).

$$
\begin{aligned}
& H_{v}(s)=\frac{1}{\tau_{v} \cdot s+1}, \\
& H_{p}(s)=\frac{1}{\tau_{p} \cdot s+1} .
\end{aligned}
$$

Finally, the controller, $C$, is a PI controller, which is usually tuned assuming that the PV generator has no influence, or that it is operating at the maximum power point (i.e. $g_{t}=0$ ).

The transfer function seen by the controller, $Z_{e q}$, has to be analyzed in order to determine the stability of the proposed voltage regulation with $P_{p v}$ compensation. From Fig. 2 it can be obtained as follows:

$$
Z_{e q}=\frac{\widehat{v}_{d c}^{2}}{\widehat{P}_{c}^{*}}=\frac{Z_{c} \cdot D \cdot G_{i} \cdot D S C}{1+Z_{c} \cdot g_{t} / 2 \cdot\left(1-H_{p} \cdot D \cdot G_{i} \cdot D S C\right)} .
$$

In order to study the stability of $Z_{\text {eq }}$, the following transfer function can be studied:

$$
L=Z_{c} \cdot g_{t} / 2 \cdot\left(1-H_{p} \cdot D \cdot G_{i} \cdot D S C\right) \text {. }
$$

\section{Stability analysis for the case study}

The characteristics of the studied application are summarized in Table II. The minimum value of the total conductance, $g_{t \min }$, appears at the minimum dc voltage, $V_{d c \min }$, and can be approximated as [6]:

$$
g_{t_{\min }}=\left(g_{p v}-g_{0}\right)_{\min } \approx-\frac{I_{s c \max }}{V_{d c \min }} .
$$

The maximum value of the total conductance, $g_{t \max }$, is not as relevant for stability as $g_{t} \min$. It is approximated with the MPP current and voltage values in STC conditions as expressed in (16).

$$
g_{t_{\text {max }}}=\left(g_{p v}-g_{0}\right)_{\text {max }} \approx 10 \cdot \frac{I_{M P P, S T C}}{V_{M P P, S T C}} .
$$

\begin{tabular}{|c|c|c|c|}
\hline Inverter rated power & $830 \mathrm{kVA}$ & Switching frequency, $f_{s w}$ & $3 \mathrm{kHz}$ \\
\hline Line to line grid rms voltage & $300 \mathrm{~V}$ & Voltage sampling time, $T_{s}$ & $1 \mathrm{~ms}$ \\
\hline Grid frequency & $50 \mathrm{~Hz}$ & DSC filter period, $T_{D S C}$ & $5 \mathrm{~ms}$ \\
\hline Maximum dc current, $I_{s c, \max }$ & $2000 \mathrm{~A}$ & Time constant of the dc voltage filter, $\tau_{v}$ & $169 \mu \mathrm{s}$ \\
\hline Minimum dc voltage, $V_{d c, \min }$ & $440 \mathrm{~V}$ & Time constant of the power filter, $\tau_{p}$ & $169 \mu \mathrm{s}$ \\
\hline Minimum total conductance, $g_{t, \min }$ & $-4.5 \mathrm{~S}$ & Crossover frequency for the current loop & $200 \mathrm{~Hz}$ \\
\hline Maximum total conductance, $g_{t, m a x}$ & $39 \mathrm{~S}$ & Phase margin for the current loop & $45^{\circ}$ \\
\hline PV generator open-circuit voltage in STC & $589 \mathrm{~V}$ & Input capacitance, $C_{d c}$ & $12.5 \mathrm{mF}$ \\
\hline PV generator MPP voltage in STC & $480 \mathrm{~V}$ & Crossover frequency for the voltage loop, $f_{c}$ & $7.5 \mathrm{~Hz}$ \\
\hline PV generator MPP current in STC & $1895 \mathrm{~A}$ & Phase margin for the voltage loop & $75^{\circ}$ \\
\hline Inverter inductance, $L_{i n v}$ & $70 \mu \mathrm{H}$ & Gain of the voltage PI controller, $K_{P}$ & 0.2949 \\
\hline Filter capacitor, $C_{f}$ & $780 \mu \mathrm{F}$ & Time constant of the voltage PI controller, $\mathrm{T}_{n}$ & 0.9385 \\
\hline Grid inductance, $L_{g}$ & $52 \mu \mathrm{H}$ & \multicolumn{3}{r}{}
\end{tabular}

Table II. Parameters of the case study. 
With the characteristics from Table II, the transfer function $L$ can be obtained. The Bode plots of $L$ for different values of $g_{t}$ are depicted in Fig. 3. The Generalized Bode Criterion (GBC) is applied now to determine the stability.

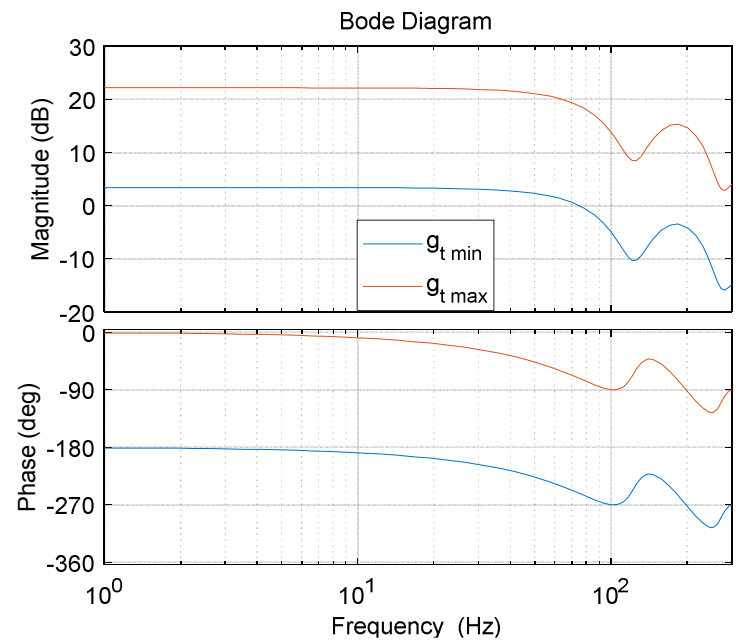

Fig. 3. Bode diagram of the transfer function $L$ expressed in (14).

For the maximum total conductance, $g_{t \max }$, the transfer function $L$ does not have poles in the right hand plane. Thus, $P=0$. From the Bode plot in Fig. 3, there are no crossings with $180^{\circ}$ with gain higher than $0 \mathrm{~dB}$, i.e. $C^{+}=C^{-}=0$. From Table I, $C_{0}=0$, since the open loop does not have poles at origin (i.e. $k=0$ ) and has a dc gain higher than -1 (i.e. $K_{G}>-1$ ). Therefore, the GBC expressed in (2), determines that $Z_{e q}$ does not have RHP poles and a stable $v_{d c}^{2}$ regulation can be obtained.

For the minimum total conductance, $g_{t \min }, L$ has neither poles in the RHP (i.e. $P=0$ ) nor crossings (i.e. $C^{+}=C^{-}=0$ ). However, from Table I, now $C_{0}=-1$ since the open loop does not have poles at origin (i.e. $k=0$ ) but has a de gain lower than -1 (i.e. $K_{G}<-1$ ) with decreasing phase at $0^{+} \mathrm{Hz}$. Applying (2), $Z_{e q}$ has one RHP pole and the $v_{d c}^{2}$ regulation will be unstable.

The PI controller of the $v_{d c}^{2}$ regulation is designed assuming that the PV generator is operating in MPP, which corresponds to $g_{t}=0$. The design parameters are a crossover frequency of $7.5 \mathrm{~Hz}$ and a phase margin of $75^{\circ}$. The resulting open loop bode plot is depicted in Fig. 4.

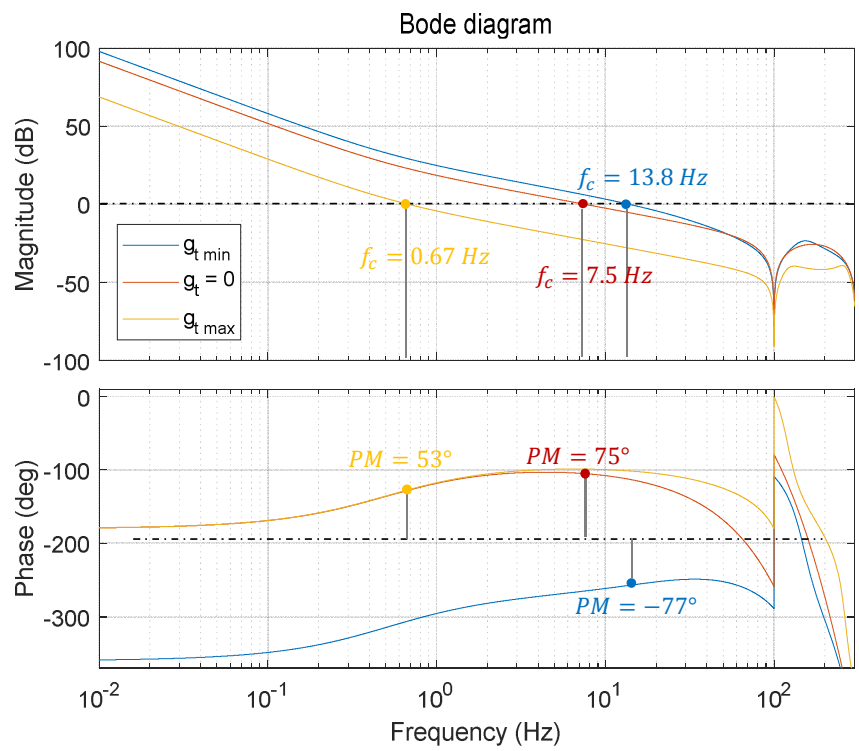

Fig. 4. Bode diagram of the open-loop $v_{d c}^{2}$ regulation for different values of the total conductance. 
The stability of the $v_{d c}^{2}$ regulation is analyzed with the GBC. The open loop does not have RHP poles for $g_{t} \max$ and $g_{t}=0$, but it has one RHP pole for $g_{t \min }$ due to $Z_{e q}$. From the Bode diagram in Fig. 4 , there are no crossings with gain higher than $0 \mathrm{~dB}$ neither for $g_{t \max }$, nor for $g_{t}=0$ nor for $g_{t \text { min. }}$ Then, both for $g_{t \max }$ and $g_{t}=0$, the open loop has two integrators, positive dc gain and increasing phase. Thus, according to Table I, $C_{0}=0$. For $g_{t \min }$, the open loop has also two integrators but negative dc gain. Therefore, from Table I, $C_{0}=-1$. The Generalized Bode Criterion expressed in (2) determines a stable $v_{d c}{ }^{2}$ regulation for $g_{t \max }$ and $g_{t}=0$, since $Z=0$. However, for $g_{t \min }$, (2) determines an unstable $v_{d c}{ }^{2}$ regulation since $Z=2$.

The PV generator and the three-phase inverter with the voltage regulation are simulated with the PSIM software. Downward steps are included in the voltage reference from open-circuit to below MPP. The results are shown in Fig. 5. It can be seen that the system is stable for $g_{t \max }$ and $g_{t}=0$, but unstable for $g_{t \min }$, as predicted with the Generalized Bode Criterion (GBC). Therefore, the voltage regulation must be modified in order to maintain a stable operation in the whole operation range.
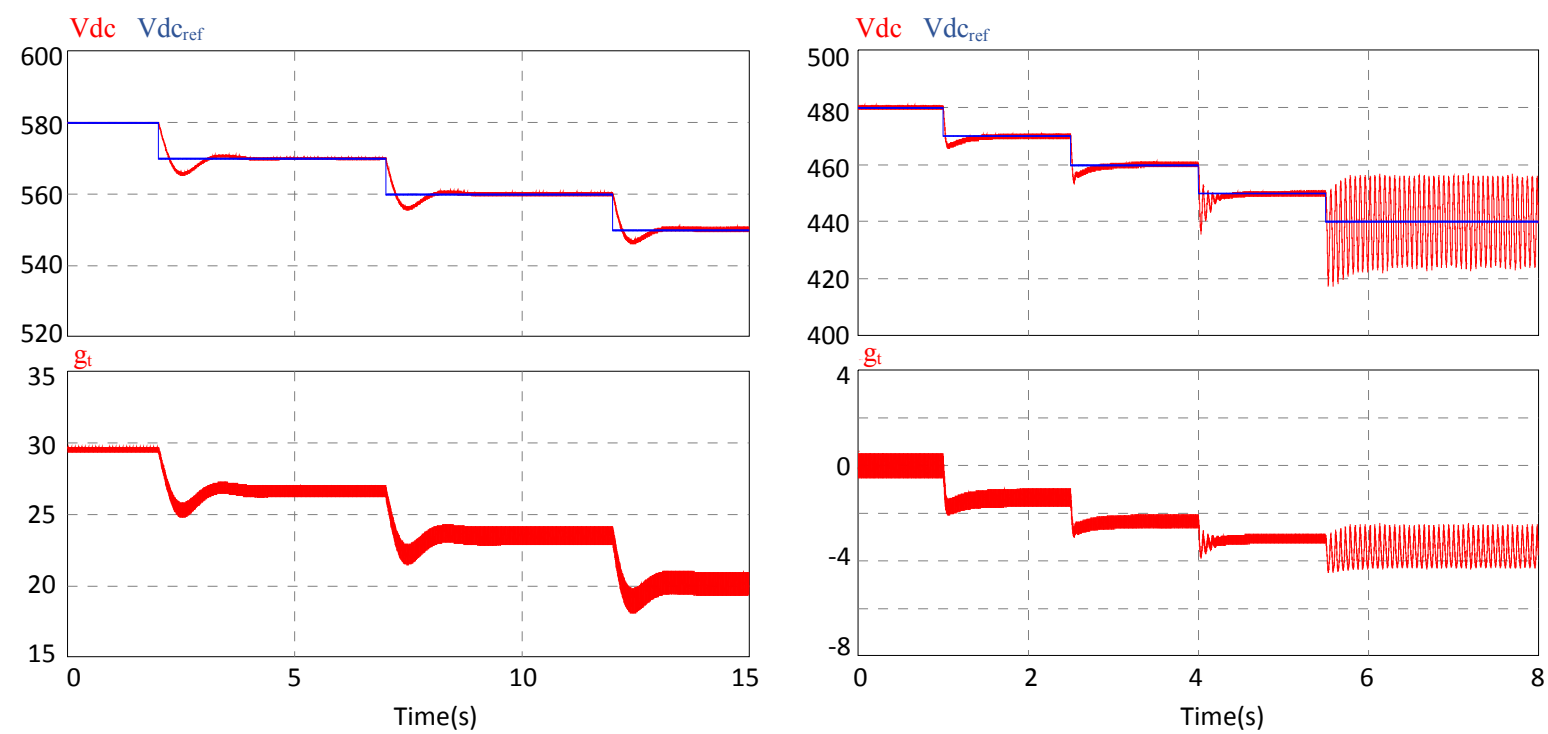

Fig. 5. Simulation results of the $v_{d c}^{2}$ voltage regulation.

The GBC can be used during the control design in order to make the system stable also for $g_{t \min }$. For this purpose, (2) is analyzed in order to be fulfilled. First, it is clear that the number of poles in the RHP of $L$ is equal to 0 and cannot be changed. Then, as the difference between $C^{+}$and $C^{-}$is going to be always an integer, to obtain a stable system, $C_{0}$ must be or zero or an even value [2]. According to Table I, it can be determined that in order to change the value of $C_{0}$ from -1 to 0 , if the number of integrators is maintained, the gain at $0 \mathrm{~Hz}$ should be changed from $K_{G}<-1$ to $K_{G}>-1$. Thus, the gain at $0 \mathrm{~Hz}$ of the transfer function in (14) is studied. It can be calculated as:

$$
L(s=0)=K_{G}=\frac{g_{t}}{C_{d c}} \cdot\left(\tau_{p}+1.5 \cdot T_{s}+2 \cdot \frac{\xi}{\omega_{n}}+\frac{5}{2} \cdot T_{s}\right),
$$

where $\tau_{p}$ corresponds to the time constant of the power filter, $1.5 \cdot T_{s}$ to the computation, digital sampling and $\mathrm{ZOH}$ delays, $2 \cdot \frac{\xi}{\omega_{n}}$ to the poles of the second order approximation of the current control loops, and $5 / 2 \cdot T_{s}$ to delay of the $D S C$.

Consequently, the gain at $0 \mathrm{~Hz}$ depends on the contribution of the poles of the transfer function in (14), the input capacitor, $C_{d c}$, and the total conductance, $g_{t}$. For $g_{t \min }$, equation (17) leads to $K_{G}=-1.65$, which again shows why the control becomes unstable. 
From (17), to increase the gain at $0 \mathrm{~Hz}$ from $K_{G}<-1$ to $K_{G}>-1$ in order to change the value of $C_{0}$ from $C_{0}=-1$ to $C_{0}=0$, the contribution of the poles could be reduced. The terms $1.5 \cdot T_{s}$ and $5 / 2 \cdot T_{s}$ are the most relevant ones in (17). However, it is not possible to reduce the existing delays in the control loop. Therefore, the only alternative to obtain $K_{G}>-1$ is by the addition of zeros to the studied transfer function. For this purpose, a lead compensator, $L E$, is now included after $P_{p v, f}$ and the modified voltage regulation is depicted in Fig. 6.

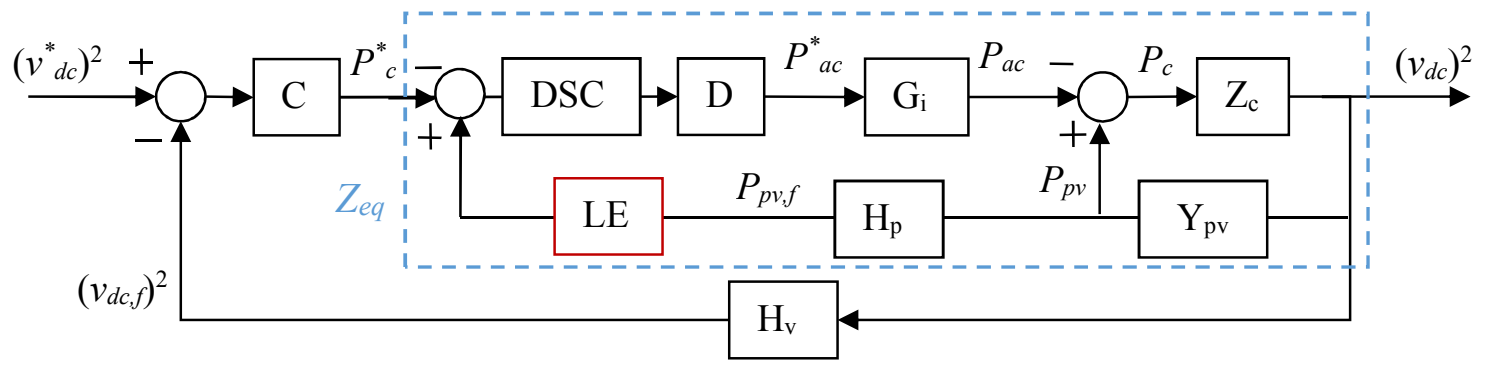

Fig. 6. Control loop for the studied $v_{d c}^{2}$ voltage regulation with lead compensator.

The transfer function of the lead compensator is expressed in (18), where $\tau_{z}$ has to be correctly chosen. The transfer function in (18) models in s-domain the lead compensator implemented in the microcontroller, which includes a pole with a time constant of $T_{s} / 2$.

$$
L E(s)=\frac{\tau_{z} \cdot s+1}{\frac{T_{s}}{2} \cdot s+1},
$$

The addition of this lead compensator to the $v_{d c}{ }^{2}$ regulation modifies the equivalent impedance seen by the controller, as Fig. 6 demonstrates. The transfer function which can be studied to obtain stability information is now transformed to:

$$
L_{L E}=Z_{c} \cdot g_{t} / 2 \cdot\left(1-L E \cdot H_{p} \cdot D \cdot G_{i} \cdot D S C\right) .
$$

The design constraint for $\tau_{z}$ is obtained by imposing to the transfer function in (19) a dc gain higher than -1 (i.e. $K_{G}>-1$ ). According to the GBC, this is the necessary condition to obtain $C_{0}=0$ and therefore a stable control loop. It is expressed as:

$$
L_{L E}(s=0)=K_{G}=\frac{g_{t}}{C_{d c}} \cdot\left(\tau_{p}+1.5 \cdot T_{s}+2 \cdot \frac{\xi}{\omega_{n}}+\frac{5}{2} \cdot T_{s}+\frac{T_{s}}{2}-\tau_{z}\right)>-1 .
$$

From (20), for $g_{t \min }, \tau_{z}>2.3 \mathrm{~ms}$ is obtained. A value of $\tau_{z}=2.5 \mathrm{~ms}$ is chosen in order to have a certain stability margin. The Bode plot of the transfer function $L_{L E}$ for the different values of the total conductance is depicted in Fig. 7.

Again, the GBC is used for the stability analysis. For $g_{t \min }$, the number of open-loop RHP poles of $L_{L E}$ is still 0 (i.e. $P=0$ ). Although a crossing with $-540^{\circ}$ appears in the Bode diagram of Fig. 7 , as it is with gain lower than $0 \mathrm{~dB}$, it is not counted. Therefore, the number of crossings also remains the same (i.e. $C^{+}=C^{-}=0$ ). However, $C_{0}$ is now 0 since the open loop does not have integrators (i.e. $k=0$ ) and has gain higher than -1 (i.e. $K_{G}>-1$ ), which was the design constraint for $\tau_{z}$. In consequence, $Z_{e q}$ does not have RHP poles for $g_{t \min }$ and a stable $v_{d c}{ }^{2}$ regulation can be obtained. For $g_{t \max }$, the stability information is the same as for the previous case without the lead compensator. 


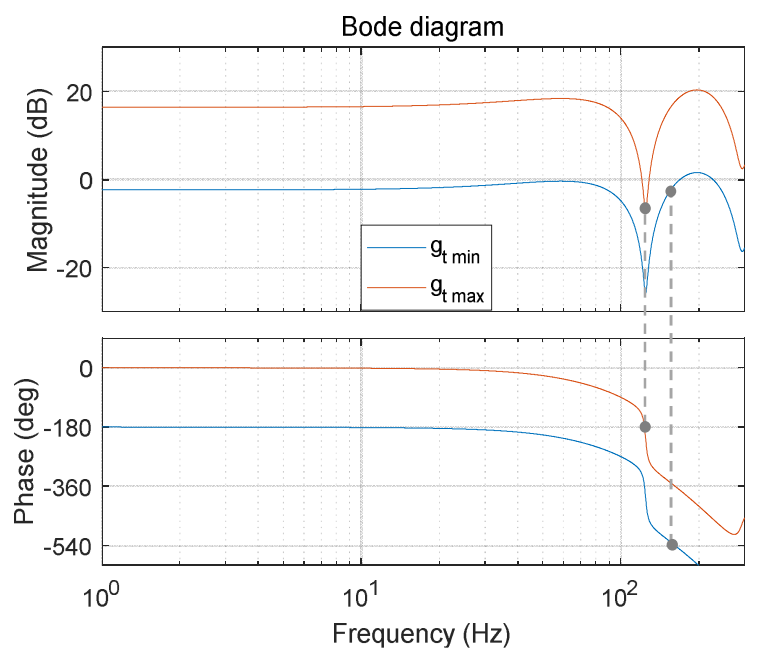

Fig. 7. Bode diagram of the transfer function $L_{L E}$ expressed in (19).

The open-loop Bode plot of the $v_{d c}^{2}$ regulation is depicted in Fig. 8. The PI controller is now designed for the case of $g_{t \min }$ and not for $g_{t}=0$. The design parameters are a crossover frequency of $22 \mathrm{~Hz}$ and a phase margin of $35^{\circ}$. Nevertheless, due to the variation of the total conductance, each point of the $P-V$ curve has a different phase margin and crossover frequency.
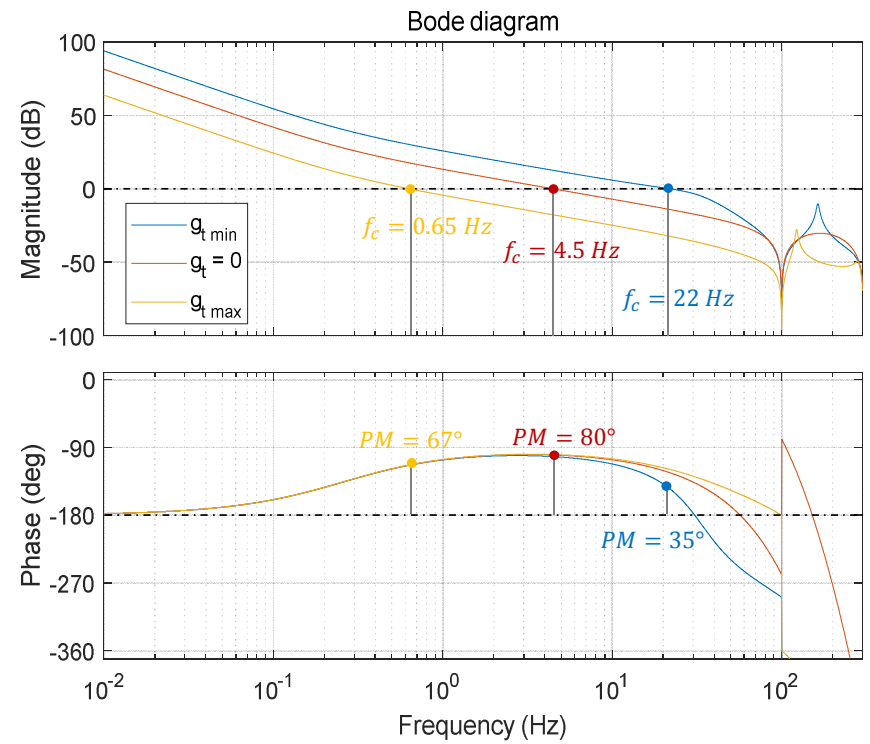

Fig. 8. Bode diagram of the modified $v_{d c}^{2}$ regulation for different values of the total conductance.

The stability of the $v_{d c}{ }^{2}$ regulation is again analyzed with the GBC. Now, the information is the same for all cases of the total conductance. The open loop does not have RHP poles. From the Bode diagram in Fig. 8, there are no crossings with gain higher than $0 \mathrm{~dB}$. Finally, the open loop has two integrators, positive dc gain and increasing phase. Thus, according to Table I, $C_{0}=0$. The Generalized Bode Criterion expressed in (2) determines a stable $v_{d c}{ }^{2}$ regulation for the whole range of the total conductance.

The simulation results of the $v_{d c}{ }^{2}$ regulation with lead compensator are shown in Fig. 9 and demonstrate that it is still stable for $g_{t \max }$ and that it is now also stable for $g_{t \min }$, thanks to the lead compensator. These results are in line with the information obtained with the Generalized Bode Criterion and demonstrate the easy application of this criterion not only for the stability analysis but also for the control design. 

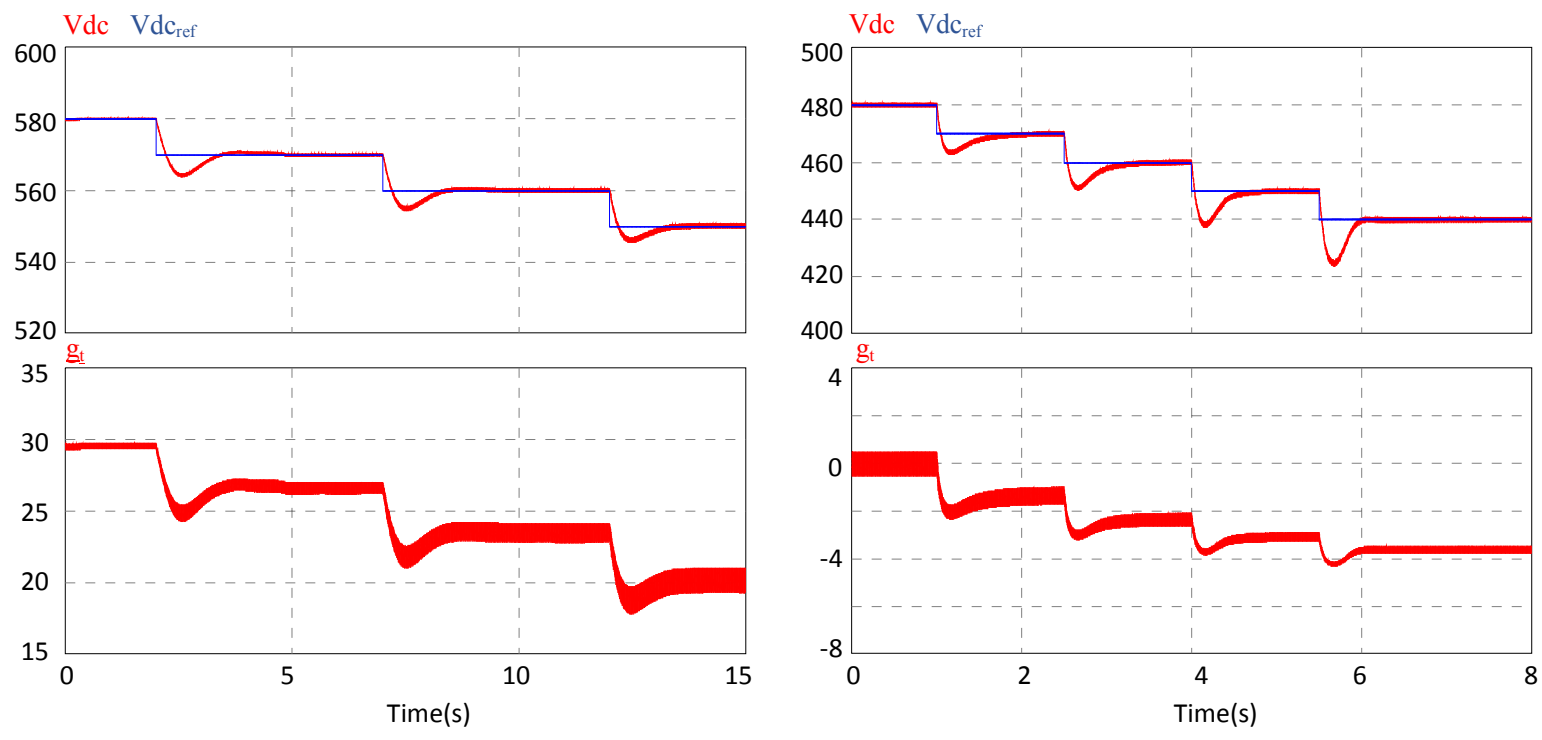

Fig. 9. Simulation results of the $v_{d c}^{2}$ regulation with lead compensator.

\section{Conclusions}

This paper analyzes the dc voltage regulation for three-phase photovoltaic grid-tied inverters where the square of the dc voltage, $v_{d c}{ }^{2}$, is controlled and a compensation of the photovoltaic power is included. The application is used to show the potential of the Generalized Bode Criterion (GBC) to correctly determine the stability of a control loop and to help to the controller design process. In this way, with the GBC, it is first identified that the voltage regulation is unstable for the minimum total conductance of the PV generator, $g_{t \min }$. Second, the $v_{d c}{ }^{2}$ regulation is modified by the addition of a lead compensator, which is designed using the GBC. The final voltage regulation with lead compensator is stable for the whole operating range, as both the $\mathrm{GBC}$ and the simulation results demonstrate.

\section{References}

[1] REN21, "Ren21: Renewables 2018 global status report," 2018. [Online]. Available: http://www.ren21.net/status-of-renewables/global-status-report/.

[2] D. Lumbreras, E. L. Barrios, A. Urtasun, A. Ursua, L. Marroyo, and P. Sanchis, "On the Stability of Advanced Power Electronic Converters: the Generalized Bode Criterion," IEEE Trans. Power Electron., no. (Early Acces).

[3] A. G. Yepes, S. Member, F. D. Freijedo, Ó. López, and J. Doval-gandoy, “Analysis and Design of Resonant Current Controllers for Voltage-Source Converters by Means of Nyquist Diagrams and Sensitivity Function,” IEEE Trans. Power Electron., vol. 58, no. 11, pp. 5231-5250, 2011.

[4] Y. Liao and X. Wang, "General Rules of Using Bode Plots for Impedance-Based Stability Analysis," in 2018 IEEE 19th Workshop on Control and Modeling for Power Electronics (COMPEL), 2018, pp. 1-6.

[5] A. Urtasun, P. Sanchis, and L. Marroyo, "Adaptive voltage control of the DC/DC boost stage in PV converters with small input capacitor," IEEE Trans. power Electron., vol. 28, no. 11, pp. 5038-5048, 2013.

[6] T. Messo, J. Jokipii, J. Puukko, and T. Suntio, "Determining the value of DC-link capacitance to ensure stable operation of a three-phase photovoltaic inverter," IEEE Trans. Power Electron., vol. 29, no. 2, pp. 665-673, 2014.

[7] H. Nyquist, "Regeneration theory," Bell Labs Tech. J., vol. 11, no. 1, pp. 126-147, 1932.

[8] M. Vidyasagar, R. K. Bertschmann, and C. S. Sallaberger, "Some simplifications of the graphical Nyquist criterion,” IEEE Trans. Automat. Contr., vol. 33, no. 3, pp. 301-305, 1988.

[9] J. Samanes, A. Urtasun, E. Gubia, and A. Petri, "Robust multisampled capacitor voltage active damping for grid-connected power converters,” Int. J. Electr. Power Energy Syst., vol. 105, pp. 741-752, 2019. 